\title{
Off-label use of an adjustable gastric banding system for pulmonary artery banding
}

\author{
Younes Boudjemline, MD, ${ }^{a, b}$ Emmanuelle Pineau, MD, ${ }^{c}$ Caroline Bonnet, MD, ${ }^{d}$ Alix Mollet, MD, ${ }^{a}$ Sylvia Abadir, MD, ${ }^{a}$ \\ Damien Bonnet, MD, ${ }^{\mathrm{a}, \mathrm{b}}$ Daniel Sidi, MD, ${ }^{\mathrm{a}, \mathrm{b}}$ and Gabriella Agnoletti, MD
}

From the aAP-HP, Hôpital Necker Enfants Malades, Service de Cardiologie Pédiatrique, Paris, France; 'bMIU 0016, Pr Lafon, Faculte de Necker, Paris, France; ${ }^{\mathrm{c} C}$ ardiothoracic Unit, Centre Chirurgical Marie Lannelongue, Le Plessis Robinson, France; ${ }^{\mathrm{d}}$ Service de Cardiologie, Centre Hospitalier de Dijon, Dijon, France.

The Assistance Publique des Hôpitaux de Paris (Paris, France) provided financial support (grant 2004/401).

Received for publication Oct 6, 2005; accepted for publication Dec 1, 2005.

Address for reprints: Younes Boudjemline, MD, Service de Cardiologie Pédiatrique, Hôpital Necker-Enfants-Malades, 149, rue de Sèvres, 75015 Paris cedex, France (E-mail: younes.boudjemline@nck.ap-hop-paris.fr).

J Thorac Cardiovasc Surg 2006;131:1130-5

$0022-5223 / \$ 32.00$

Copyright (๑) 2006 by The American Association for Thoracic Surgery

doi:10.1016/j.jtcvs.2005.12.045
Background: Pulmonary artery banding is proposed as a first palliation in infants with complex congenital heart disease and high pulmonary blood flow. In addition, it may be used to retrain the left ventricle. Optimal tightening may be difficult to obtain, leading to reoperation. An implantable device for pulmonary artery banding with telemetric control was recently developed allowing for repeated adjustments, but it is presently limited to patients weighing less than $20 \mathrm{~kg}$. In large animals, we tested an off-label adjustable gastric banding system for pulmonary artery banding.

Methods and Results: Fourteen ewes weighing 50 to $75 \mathrm{~kg}$ underwent implantation of the Lap-Band device (BioEnterics Corp, Santa Barbara, Calif) around the main pulmonary artery through a left thoracotomy. All had functional evaluation with progressive occlusion and opening of the device at implantation and every 2 weeks until sacrifice immediately after implantation (group $1, \mathrm{n}=8$ ), at 1 month (group $2, \mathrm{n}=3$ ), at 3 months (group 3, $\mathrm{n}=3$ ), or death. Invasive pressure measurements in the right ventricle and aorta were carried out each time. Devices were easily implanted in all animals. Progressive occlusion and reopening were possible in all animals during each time point. Two animals died of right heart failure related to excessive tightening of the band. Retrieval of the device without any major damage was possible in 12 of 14 animals.

Conclusion: With this implantable device, we were able to adjust the pulmonary artery diameter in animals. Patients requiring left ventricle retraining and weighing more than $30 \mathrm{~kg}$ would benefit from the device's use in humans.

I n patients with corrected transposition of the great arteries, the double-switch operation is a surgical option, but left ventricle pumping in the pulmonary circulation needs to be retrained to assume the systemic work load. ${ }^{1-3}$ Pulmonary artery banding (PAB) has been used to increase left ventricular afterload in such situations. ${ }^{4,5}$ Tightening of the band is problematic, and some patients require a PAB reoperation because of excessive tightening, leading to left ventricular dysfunction, or insufficient tightening with inappropriate ventricular preparation. These reoperations are associated with increased morbidity and mortality. In these patients, a dilatable banding system is of interest. To date, only 1 adjustable device (ie, the FlowWatch, EndoArt S.A., Lausanne, Switzerland) is presently available, but its use is limited to small children weighing less than $20 \mathrm{~kg} .{ }^{6-9}$ The Lap-Band adjustable banding system (BioEnterics Corp, Santa Barbara, Calif) was initially designed to induce a restriction to the gastric diameter in severely obese patients. ${ }^{10-12}$ We investigated the off-label use of this device to create an adjustable $\mathrm{PAB}$. We report here the preliminary experimental application.

\section{Methods and Materials \\ Device Description}

The Lap-Band adjustable banding system is a 13-mm-wide band that, when fastened, forms a circular ring with an inside circumference of $9.75 \mathrm{~cm}$ (Figure 1). The band is made of 


\section{Abbreviations and Acronyms \\ PA = pulmonary artery \\ $\mathrm{PAB}=$ pulmonary artery banding}

biocompatible silicon elastomers. The inner surface of the band is inflatable and connected by 50-cm kink-resistant tubing to an access port placed subcutaneously. The tubing might be shortened to tailor the position of the port to the size of animals. A locking mechanism is located around the ring to avoid undesired opening of the band during inflation. The device is available in different sizes. For this application, we used the smallest commercially available device with a circumference of $9.75 \mathrm{~cm}$. When the device is clipped, the dimensions are $13 \mathrm{~mm}$ (width), $26 \mathrm{~mm}$ (internal diameter), and $38 \mathrm{~mm}$ (external diameter). The adjustable area in a fully open position corresponds to a pulmonary artery (PA) diameter of $26 \mathrm{~mm}$ and is fully closed after injection of $10 \mathrm{~mL}$ of saline, theoretically allowing for PAB in adolescents and adults (Figure 2). No energy is required to reduce the diameter of the device. The change in the adjustable area is obtained by saline injection in the inner surface of the band from the access port along the tubing.

\section{Animal Preparation}

Fourteen ewes weighing 55 to $75 \mathrm{~kg}$ were included in the present study and divided into 3 groups according to the time of sacrifice. Eight animals were sacrificed acutely (group 1), whereas the remaining 6 were kept alive for 1 month (group 2) and 3 months (group 3). Acute group was performed to evaluate (1) the applicability of the off-label use of the device around the PA and (2) the possibility of progressively narrowing and releasing the PA with balloon inflation and deflation. In groups 2 and 3, we tested the function of the device up to 12 weeks after its implantation, with repeated adjustments of PAB. All animals received medical care according to the European Guidelines for Laboratory Animal Care. $^{13}$

Insertion of the Adjustable Pulmonary Artery Banding Ewes were sedated with an intravenous injection of pentothal (10 $\mathrm{mg} / \mathrm{kg}$ body weight), placed in a lateral position, intubated, and ventilated with $100 \%$ oxygen and $1 \%$ halothane. Anesthesia was maintained with halothane $(0.5 \%-3 \%)$. Cardiac and respiratory functions were monitored throughout the procedure. A left thoracotomy was performed in the fourth intercostal space. The pericardium was longitudinally opened. The main PA was dissected posteriorly. To avoid the risk of secondary migration, the dissection was limited to the space needed to insert the system $(\sim 1.5 \mathrm{~cm})$. A device was opened, straightened, and placed around the main PA. Particular attention was taken not to traumatize the vessel and surrounding structures when passing the system around the main PA. The locking mechanism of the device was left anteriorly after closure of the device to test the possibility of retrieval. The device was not sutured to the main PA. After the evaluation (see below), animals were sacrificed (acute study, group 1) or extubated (chronic study, groups 2 and 3). Drains were removed 2 to 3 hours after the animals were completely awake. Postoperative medical treatment consisted of antibiotics for 48 hours and analgesia.

\section{Hemodynamic Assessment}

After the device was inserted, all ewes underwent hemodynamic evaluation consisting of invasive measurements of right ventricular, PA, and aortic pressures. Pressures were measured after sequential inflations of saline in the access port connected to the band. Pressure catheters were inserted into the right ventricle after its direct puncture and into the distal PA for simultaneous pressure monitoring. An arterial femoral access was obtained to assess systemic blood pressure. For long-term study, the ventricular catheter was fixed to the right ventricle with a 3-0 nonresorbable thread. The extremity of the catheter was connected to an access port placed and fixed subcutaneously near the device port. The catheter was flushed with heparin to limit the risk of occlusion. At the time of control, femoral artery access was gained for systemic blood pressure control, and 2 access ports (RV catheter and device) were punctured with two 22-gauge needles. Pressures were recorded simultaneously. Maximal inflation volume leading to abrupt systemic pressure drop and its corresponding right ventricular pressure were first determined. Various inflations were then made with a decrement of $0.2 \mathrm{~mL}$ from the maximal inflation volume until normalization of the right ventricular pressure. The minimal inflation volume was determined and defined as the volume of saline leading to normalization of right ventricular pressure. Smaller decrements and increments of $0.1 \mathrm{~mL}$ were necessary to determine precisely the minimal inflation volume. Measure-

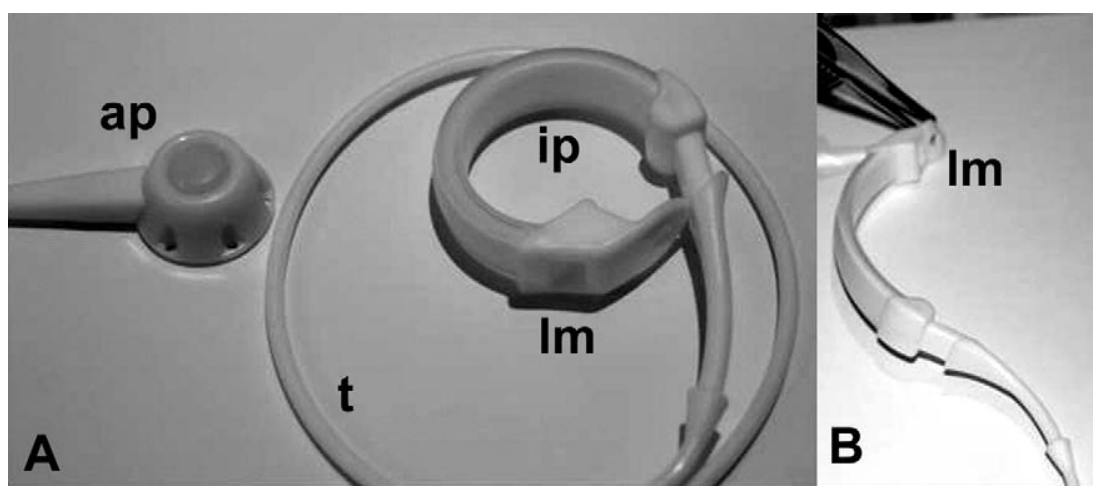

Figure 1. Band in closed (A) and opened (B) positions. ap, Access port; ip, inflatable part; Im, locking mechanism; $t$, kink-resistant tubing. 


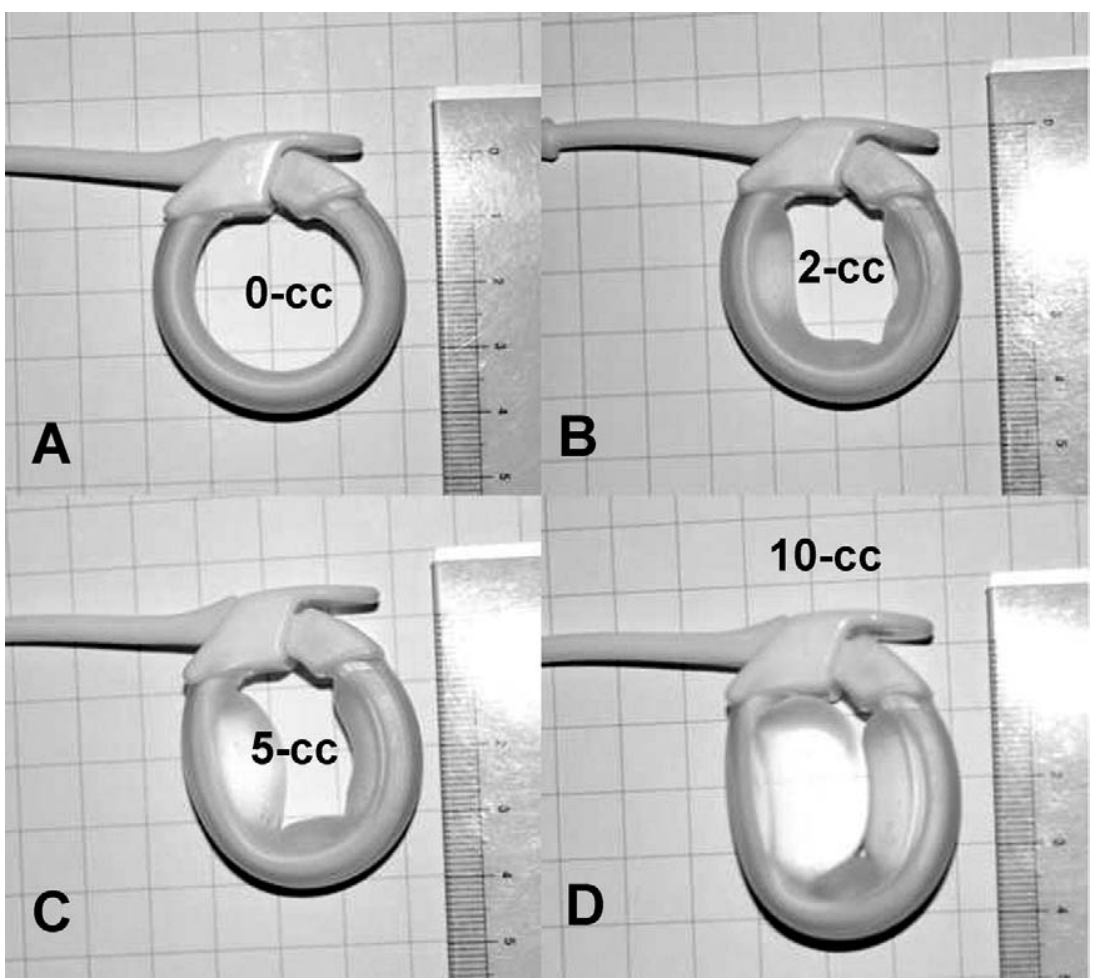

Figure 2. Band in different degrees of inflation. A, Steady state. B, After inflation of $2 \mathrm{~mL}$ of saline. C, Configuration after inflation of 5 $\mathrm{mL}$ of saline. D, Complete closure of the band (10 mL).

ments were made after 2 minutes to obtain a steady state. A period of 5 minutes was respected between inflations to achieve complete recovery of systemic pressure and return to baseline conditions.

\section{Follow-up of Animals in Groups 2 and 3}

For long-term observation, ewes were transferred to a farm. Animals were reassessed weekly in group 2 (weeks 1, 2, 3, and 4) and every 2 weeks in group 3 (weeks 2, 4, 6, 8, 10, and 12). The assessment was performed sooner if any symptoms occurred. Reevaluation consisted of a hemodynamic assessment under general anesthesia. We expected to leave the band inflated at the end of each reassessment with a saline volume of minimum inflation volume plus $0.3 \mathrm{~mL}$. This inflation volume was recorded and compared with the saline volume to full deflation at the beginning of each control to assess the porosity of the device.

\section{Graft Retrieval}

All grafts were explanted at the end of the protocol (ie, acutely in group 1, after 1 month in group 2, and after 3 months in group 3 ). In group 1, devices were retrieved with a beating heart to assess the easiness of retrieval. Elsewhere, the heart and lungs were retrieved together in 1 block. All hearts were dissected and inspected macroscopically. The pulmonary vascular tree was examined to determine the position of each device in relation to adjacent structures. The possibility of device retrieval was studied in groups 2 and 3 .

\section{Results}

Animals in Group 1

The acute experimental study confirmed the easy insertion of the device around the main PA (8/8) without any damage to the surrounding structures (Figure 3). The invasive pressure assessment showed the appearance of a pressure gradient between the proximal and distal part of the adjustable band. This gradient increased progressively in parallel with

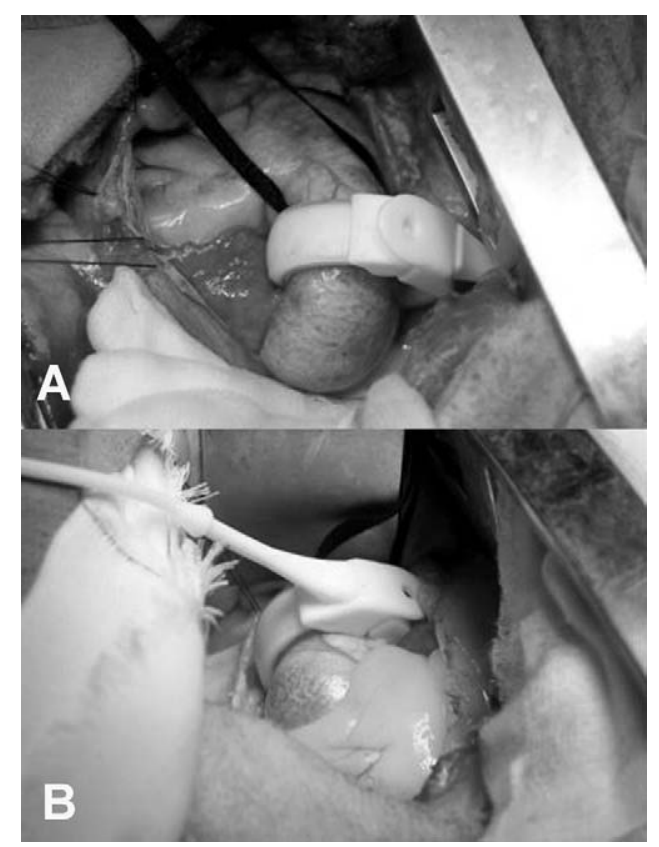

Figure 3. Surgical views showing the band after its placement around the main pulmonary artery. 
TABLE 1. Data from animals in group 1 (acute study)

\begin{tabular}{cccc}
\hline $\begin{array}{c}\text { Animal } \\
\text { number }\end{array}$ & $\begin{array}{c}\text { MaxInfVol/ } \\
\text { MaxRVP } \\
(\mathbf{m L} / \mathbf{m m ~ H g})\end{array}$ & $\begin{array}{c}\text { MinlnfVol }+ \\
\mathbf{0 . 3} \mathbf{~ m L / R V P ~} \\
(\mathbf{m L} / \mathbf{m m ~ H g})\end{array}$ & $\begin{array}{c}\text { MinlnfVol/ } \\
\text { MinRVP } \\
(\mathbf{m L} / \mathbf{m m ~ H g})\end{array}$ \\
\hline 1 & $6.3 / 90$ & $5.3 / 30$ & $5.0 / 20$ \\
2 & $5.9 / 65$ & $5.1 / 35$ & $4.8 / 15$ \\
3 & $6.0 / 88$ & $5.3 / 25$ & $5.0 / 18$ \\
4 & $6.1 / 92$ & $5.5 / 33$ & $5.2 / 22$ \\
5 & $5.8 / 86$ & $5.2 / 26$ & $4.9 / 14$ \\
6 & $5.8 / 75$ & $5.3 / 30$ & $5.0 / 20$ \\
7 & $6.2 / 95$ & $5.3 / 24$ & $5.0 / 16$ \\
8 & $5.9 / 89$ & $5.2 / 35$ & $4.9 / 25$ \\
Mean & $6 / 85$ & $5.3 / 30$ & $5.0 / 19$ \\
Range & $5.8-6.3 / 65-95$ & $5.1-5.5 / 24-35$ & $4.8-5.2 / 14-25$ \\
\hline
\end{tabular}

The minimal inflation volume was determined and defined as the volume of saline leading to normalization of RVP. The maximal inflation volume was the volume leading to abrupt systemic pressure drop. MaxInfVol, Maximal inflation volume; MaxRVP, maximal right ventricular pressure; MinInfVol, minimal inflation volume; MinRVP, minimal right ventricular pressure.

the occlusion of the device and instillation of saline. The reopening of the device was followed by progressive reduction of the pressure gradient and right ventricular pressure. The mean maximum right ventricular pressure was $85 \mathrm{~mm}$ $\mathrm{Hg}$ (range 65-95 $\mathrm{mm} \mathrm{Hg}$ ), and the inflation volume was 6 $\mathrm{mL}$ (range 5.8-6.3 mL). Hemodynamic data are shown in Table 1. It was possible to retrieve the band in all animals before sacrifice.

\section{Animals in Groups 2 and 3}

In the acute study, all devices were implanted without any problem. Acute measurements were not different from group 1. The postoperative course was uneventful in all animals.

Reassessment was performed weekly in all group 2 animals. In group 3, only 1 animal was reassessed as planned. In the remaining 2 animals, assessments were performed as planned until week 6 after the initial procedure. Because of organizational problems, testing was delayed and performed 1 week (week 9) after the planned control (week 8).

No animals in group 2 died during the entire follow-up, whereas 2 animals in group 3 died. One animal died afterl anesthetic induction (week 9). At autopsy, signs of right ventricular failure (ie, pleural effusion and right ventricular enlargement) were found. The second animal, also tested at week 9, had a pericardial effusion $(1.5 \mathrm{~L})$ that was drained. Pressure measurements indicated right ventricular failure. Therefore, the band was left open after the procedure to allow for recovery of the right ventricle. The animal was found dead in its cage at week 10. The same features were found at autopsy. After this problem, the PAB was left open after each testing in the remaining animal in group 3; the animal had an uneventful follow-up until sacrifice at 12 weeks after device insertion.

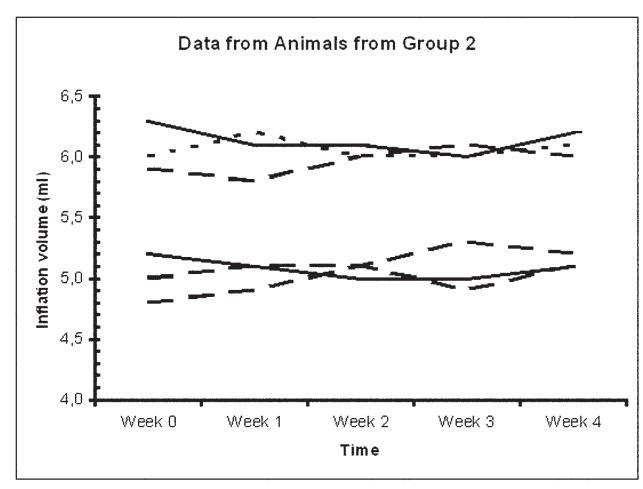

Figure 4. Data from group 2.

At the 1-week evaluation in group 2, a small skin incision was necessary in 2 animals to locate the port because entry through the access port connected to the RV pressure was not possible. It was refixed subcutaneously at the end of the study, and its access was possible at each following study. One animal had a subcutaneous infective collection that was treated by surgical evacuation and resection of infective tissue. Because the ports were left in place, a complete cure was not possible and the animal continued to experience local suppuration throughout the protocol.

Full deflation of the balloon was always possible at the beginning of the reassessment. Perfect equivalence was found when comparing the saline volume with full deflation in the beginning of each control and the inflation volume left at previous controls, reflecting the absence of fluid loss through the system.

In all animals, repeated inflation and deflation of the device were confirmed at implantation and during each testing throughout the protocol. Hemodynamic data at each control are shown in Figures 4 and 5. There was a good correlation between volume inflation and ventricular pressure increase; maximum and minimum volume inflation and pressure regimen response values were in the same range. With time, response to the same volume inflation was variable when compared individually.

Animals in group 2 were sacrificed as planned after a mean time from implantation of 4.5 weeks (range 4-5 weeks). In animals in groups 2 and 3, the device was still functioning. The device was easily identified through a thoracic incision; it was located in the same position of implantation, without any migration of the device. Before retrieval, macroscopic examination revealed that 4 of 6 devices were free of any process ( 3 from group 2 and 1 from group 3). Removal of the device was possible in those animals without any lesion to the surrounding structures, bleeding, or hemodynamic disturbance (Figure 6). The PA was not altered macroscopically. Elsewhere, fibrous tissue was present, completely surrounding the device (2 animals 


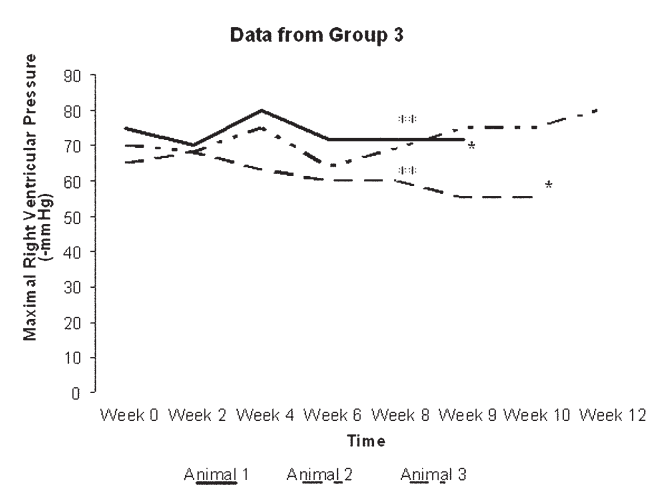

Figure 5. Data from group 3. **Not performed. *Time of death. Animal 1 died after anesthetic induction before device and pressure control at week 9. Animal 2 was found dead at week 10 in the farm.

in group 3) and making its retrieval difficult without any lesion to the PA.

\section{Discussion}

Several attempts at developing an adjustable PAB have been made, but only 1 device is commercially available. The FloWatch system is capable of repeated narrowing and releasing of the PA with a telemetric control. This device is presently used in humans, but its use is limited to small children weighing less than $20 \mathrm{~kg}$. Older patients might need an adjustable PAB. We investigated the use of an off-label adjustable device for gastric restriction. Our experimental study demonstrated that the device is easy to implant and use. Repeated narrowing and releasing of the
PA were possible in the acute and chronic study groups. Furthermore, no loss of fluid was found when comparing volume with full deflation at the beginning of testing and volume left at the end of the previous testing, reflecting the absence of porosity of the device. At the time of explantation, retrieval of the device was always possible in animals with the shortest follow-up. However, it is probable that the device will be covered by fibrous tissue making its retrieval more difficult after a long period of time, as seen in 2 animals in which the implant was left for 10 and 12 weeks. This could hamper or complicate further surgery, making plastic repair of the PA mandatory before the double-switch operation can be performed in humans. In such a population, the use of the device should probably be limited in time with rapid training. Death occurred in 2 animals from group 3 , due to right ventricular failure. Indeed, scheduled testing procedures and release of the device had to be delayed for a week because of organizational problems. When the 2 animals were tested, right ventricular failure was demonstrated; 1 animal died at anesthetic induction, and 1 animal died after 1 week despite complete release of the banding. Closer follow-up would clearly have avoided this fatal issue. One of the difficulties we encountered was the need for anesthesia to test the device. It is probable that the level of narrowing was too high, leading to heart failure in awake animals. This was not seen in animals tested weekly but in animals tested every 2 to 3 weeks. It would have been more appropriate to test the device in fully awake animals, at rest or even at exercise, to properly assess the capability of the right ventricle to cope with the increased afterload. In humans, the assessment would be easier and allow narrowing or releasing of the banding system under echocardiographic

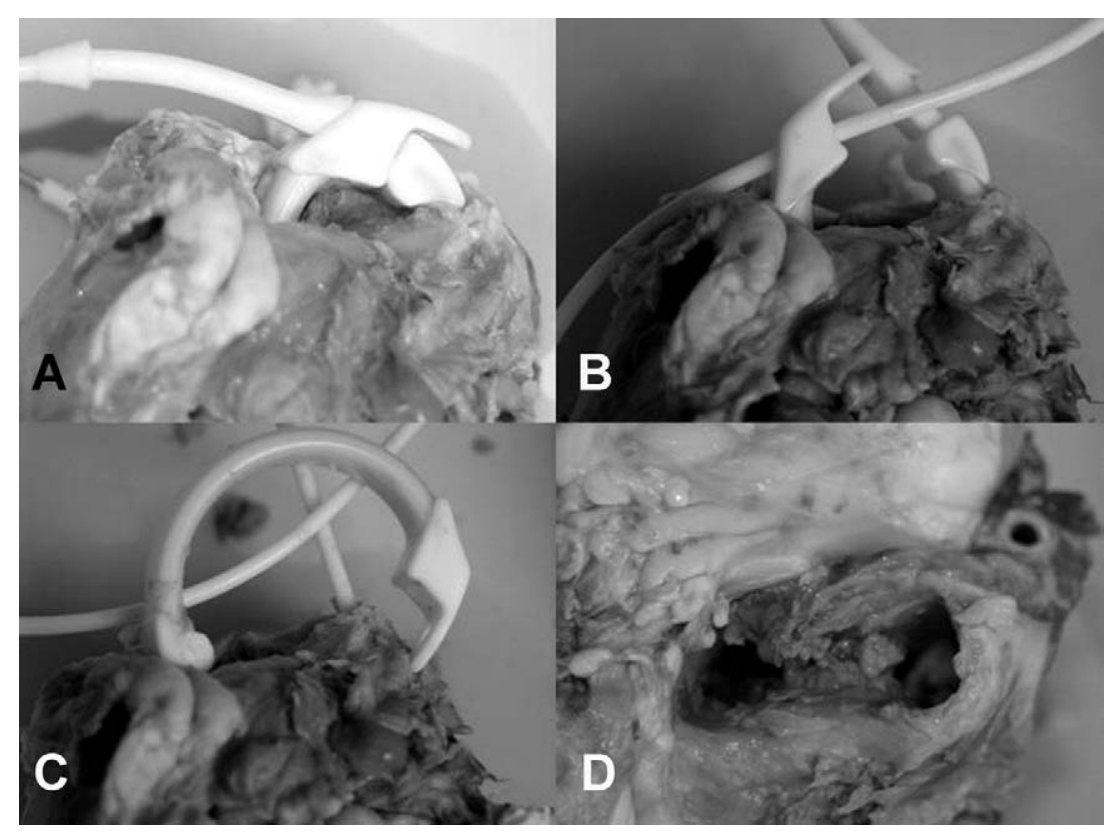

Figure 6. Anatomic pictures showing the device during its retrieval after 1 month of insertion. A, Band still around the pulmonary artery with the locking mechanism clipped. B, The locking mechanism has been opened. C, Partial retrieval of the band. D, Complete retrieval of the band. Note that complete retrieval is possible without extensive dissection.

1134 The Journal of Thoracic and Cardiovascular Surgery • May 2006 
control in nonsedated patients. Exercise could, moreover, be simulated using intravenous drugs.

Patients with a morphologic right ventricle in the systemic circulation (as seen in corrected transposition of the great arteries or previous atrial switch operation) may require a PAB for retraining of the left ventricle before anatomic biventricular repair. Whether a left ventricle can or cannot be retrainable after a certain age is still controversial. In most studies, there is a "clear" cutoff. ${ }^{1,2}$ Patients in whom left ventricular retraining is started before the age of 12 years have a greater rate of completion than older patients with the current banding protocol. The problem with this management, particularly in asymptomatic patients, is the risk of failure (ie, debanding, left ventricular failure, transplantation, and death). We are convinced that the type of retraining is crucial, as recently demonstrated by Le Bret and colleagues ${ }^{14}$ in an experimental study. It is possible that, as advocated by them, fitness defined as repeated periods of training followed by complete relapse of the afterload is preferable to a constant training that may lead to ventricular fibrosis. However, the so-called fitness technique is difficult to perform in clinical practice with conventional PA banding but would clearly be possible with the Lap-Band device. The results of left ventricle retraining with the new device may be better in an older population, in whom there is an inconsistent response to left ventricular retraining. The best way to retrain a left ventricle is still unknown. In particular, periodicity, timing, and length of training are to be defined. Another benefit of the Lap-Band device is the ability to restore the initial pressure regimen when needed. It has a dual implication. First, the postoperative course could be facilitated by the complete release of the banding with retraining started after a few days with the patient in physiologic hemodynamic conditions. Second, in case the device is not tolerated, it could be completely released and left open without requiring any reintervention for PA debanding.

\section{Conclusion}

We described the off-label use of a device capable of repeated narrowing and releasing of the PA with external control in adult animals. The preliminary experimental study demonstrated the feasibility of the implant and good function of the device up to 12 weeks after its insertion. Of course, long-term experimental studies will be required before human application, but preliminary results are promis- ing. The use of this device in humans would have tremendous benefit on the management of congenital heart diseases. The size of the currently available device will limit the clinical application to patients weighing more than $40 \mathrm{~kg}$, and therefore a smaller size will be necessary for smaller children.

The authors thank BioEnterics Corporation for providing the system and the URC of Necker Hospital for technical support.

\section{References}

1. Winlaw DS, McGuirk SP, Balmer C, Langley SM, Griselli M, Stumper O, et al. Intention-to-treat analysis of pulmonary artery banding in conditions with a morphological right ventricle in the systemic circulation with a view to anatomic biventricular repair. Circulation. 2005;111: 405-11.

2. Poirier NC, Yu JH, Brizard CP, Mee RB. Long-term results of left ventricular reconditioning and anatomic correction for systemic right ventricular dysfunction after atrial switch procedures. J Thorac Cardiovasc Surg. 2004;127:975-81.

3. LeBlanc JG, Ashmore PG, Pineda E, Sandor GG, Patterson MW, Tipple M. Pulmonary artery banding: results and current indications in pediatric cardiac surgery. Ann Thorac Surg. 1987;44:628-32.

4. Ilbawi MN, Idriss FS, DeLeon SY, Muster AJ, Gidding SS, Duffy CE, et al. Preparation of the left ventricle for anatomical correction in patients with simple transposition of the great arteries. Surgical guidelines. J Thorac Cardiovasc Surg. 1987;94:87-94.

5. Dyer K, Graham TP. Congenitally corrected transposition of the great arteries: current treatment options. Curr Treat Options Cardiovasc Med. 2003;5:399-407.

6. Fridez P, Jordan A, Montavon JC, Stergiopulos N. FloWatch: an implantable device for telemetric control of flow after pulmonary artery banding. Cardiovasc Eng. 2002;7:51.

7. Corno AF, Bonnet D, Sekarski N, Sidi D, Vouhe P, von Segesser LK. Remote control of pulmonary blood flow: initial clinical experience. J Thorac Cardiovasc Surg. 2003;126:1775-80.

8. Corno AF, Sekarski N, Bernath MA, Payot M, Tozzi P, von Segesser LK. Pulmonary artery banding: long-term telemetric adjustment. Eur J Cardiothorac Surg. 2003;23:317-22.

9. Bonnet D, Corno AF, Sidi D, Sekarski N, Beghetti M, Schulze-Neick I, et al. Early clinical results of the telemetric adjustable pulmonary artery banding FloWatch-PAB. Circulation. 2004;110:II158-63.

10. Angrisani L, Di Lorenzo N, Favretti F, Furbetta F, Iuppa A, Doldi SB, et al. The Italian Group for LAP-BAND: predictive value of initial body mass index for weight loss after 5 years of follow-up. Surg Endosc. 2004;18:1524-7.

11. Angrisani L, Favretti F, Furbetta F, Iuppa A, Doldi SB, Paganelli M, et al. Italian Group for Lap-Band System: results of multicenter study on patients with BMI $<$ or $=35 \mathrm{~kg} / \mathrm{m} 2$. Obes Surg. 2004;14:415-8.

12. Angrisani L, Furbetta F, Doldi SB, Basso N, Lucchese M, Giacomelli F, et al. Lap band adjustable gastric banding system: the Italian experience with 1863 patients operated on 6 years. Surg Endosc. 2003;17:409-12.

13. Convention Européenne sur la protection des animaux vertébrés utilisés à des fins expérimentales ou à d'autres fins scientifiques. Journal Officiel des Communautés Européennes. 1999;L222:29-37.

14. Le Bret E, Lupoglazoff JM, Borenstein N, Fromont G, Laborde F, Bachet J, et al. Cardiac "fitness" training: an experimental comparative study of three methods of pulmonary artery banding for ventricular training. Ann Thorac Surg. 2005;79:198-203. 